Research Article

\title{
Regularization Methods for Phase Retrieval and Phase Contrast Tomography
}

\author{
Bruno Sixou \\ CREATIS, INSA-Lyon, Inserm, U1044, CNRS UMR 5220, Université Lyon 1, Université de Lyon, 69621 Lyon, France \\ Correspondence should be addressed to Bruno Sixou; bruno.sixou@insa-lyon.fr
}

Received 26 September 2014; Accepted 26 December 2014

Academic Editor: Bangti Jin

Copyright (C) 2015 Bruno Sixou. This is an open access article distributed under the Creative Commons Attribution License, which permits unrestricted use, distribution, and reproduction in any medium, provided the original work is properly cited.

Phase contrast tomography is a high sensitivity medical imaging technique. Several regularization methods have been used in the literature to obtain stable solutions for the phase retrieval or the phase contrast tomography problems. Yet, the functional framework and the convergence properties of the methods have not been studied in detail. In this work, the convergence properties of regularization approaches for phase retrieval and phase contrast tomography are investigated.

\section{Introduction}

$\mathrm{X}$-ray microtomography is a very useful imaging technique in material science [1] and medical imaging [2-6]. Yet its sensitivity is improved for soft tissues within dense materials with phase contrast imaging obtained with third-generation synchrotrons. X-ray in-line phase tomography is based on coupling of tomography and phase retrieval to reconstruct the real part of the refractive index $[7,8]$. For coherent $\mathrm{X}$ rays, phase contrast can be achieved by letting the beam propagate in the free space after interaction with the object and by recording the intensity for one or several propagation distances and for several projection angles (Figure 1). The relationship between the phase shift induced by a sample and the intensity recorded at a given sample-to-detector distance relies on the Fresnel diffraction theory [9-12]. Several linear algorithms have been proposed for the phase retrieval from the Fresnel diffraction patterns [1,10,13-18] valid under some restrictive assumptions. Yet, in these works, the convergence of the regularization methods used to obtain stable solutions has not been investigated. The main purpose of this paper is to detail the functional properties of the direct and inverse operators involved in these problems on the basis of classical results of harmonic analysis. Our aim is also to study the convergence of the regularized solutions when the noise and the regularization parameter tend to zero for the phase retrieval and the phase contrast tomography problems in the good functional framework. To prove stability with respect to the noise, we have to define a parameter choice rule for $\gamma$ depending on the noise level $\delta$. This procedure is typical for regularizing ill-posed problems [19] and is presented in detail.

This paper is organized as follows. In Section 2, we present in detail the phase retrieval and phase contrast tomography direct problems. In Section 3, we consider the various regularization methods proposed in the literature for the linearized phase retrieval problem. We investigate the behaviour of the solutions as the regularization parameter tends to zero with the noise level. In Section 4, we study the convergence properties of the solutions of the phase contrast tomography problem obtained with various regularization methods. These methods are based on a combination of inversion schemes for the Radon projector and the phase operator.

\section{Phase Retrieval and Phase Contrast Tomography}

2.1. The Direct Problem for Phase Contrast Tomography. In the following, we will consider a monochromatic, coherent, parallel X-ray beam. The real and imaginary parts of the complex refractive index to be reconstructed, denoted as $\delta_{n}$ and $\beta$, are defined on a 3D bounded domain $(\Sigma)$ with spatial coordinates $(x, y, z)$. For the sake of simplicity, we assume 


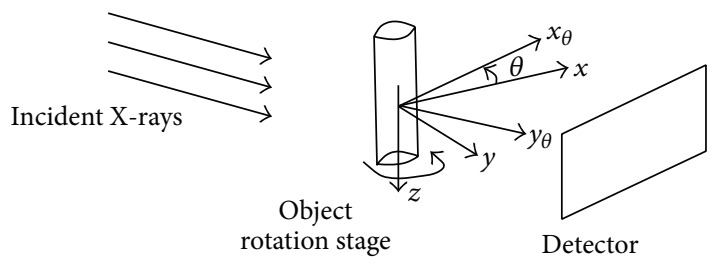

FIGURE 1: Experimental set-up in propagation based phase contrast tomography from a single propagation based phase contrast image per projection showing the coordinate system.

that $\Sigma$ is the Cartesian product $\Sigma_{x} \times \Sigma_{y} \times \Sigma_{z}$ of bounded intervals on the coordinate axes. We denote by $\left(x_{\theta}, y_{\theta}, z\right)$ the rotated spatial coordinate system for an angle $\theta$ around the $z$-axis (Figure 1).

The optical properties of an object interacting with coherent X-rays of wavelength $\lambda$ are related to its complex refractive index given by

$$
n(x, y, z)=1-\delta_{n}(x, y, z)+i \beta(x, y, z),
$$

where $\delta_{n}$ is the refractive index decrement and $\beta$ is the absorption index [20]. For a fixed projection angle $\theta$, thin objects, and straight line propagation of the beam along the $y_{\theta}$ direction, this interaction can be described by a transmittance function $T$ of the coordinates $X_{\theta}=\left(x_{\theta}, z\right)$ :

$$
\begin{aligned}
T_{\theta}\left[\delta_{n}\right]\left(X_{\theta}\right) & =\exp \left(-B_{\theta}[\beta]\left(X_{\theta}\right)+i \varphi_{\theta}\left[\delta_{n}\right]\left(X_{\theta}\right)\right) \\
& =a_{\theta}\left(X_{\theta}\right) \exp \left(i \varphi_{\theta}\left[\delta_{n}\right]\left(X_{\theta}\right)\right),
\end{aligned}
$$

where $a_{\theta}[\beta]\left(X_{\theta}\right)$ is the absorption and $\varphi_{\theta}\left[\delta_{n}\right]\left(X_{\theta}\right)$ is the phase shift induced by the object for the projection angle $\theta$ [8]. The phase and the negative logarithm of the absorption, $B_{\theta}[\beta]\left(X_{\theta}\right)=-\log \left(a_{\theta}[\beta]\left(X_{\theta}\right)\right)$, are the projections of the absorption index and refraction index, respectively:

$$
\begin{gathered}
B_{\theta}[\beta]\left(X_{\theta}\right)=\frac{2 \pi}{\lambda} \int \beta\left(y_{\theta}, X_{\theta}\right) d y_{\theta}, \\
\varphi_{\theta}\left[\delta_{n}\right]\left(X_{\theta}\right)=\frac{2 \pi}{\lambda} \int\left(1-\delta_{n}\left(y_{\theta}, X_{\theta}\right)\right) d y_{\theta},
\end{gathered}
$$

where $y_{\theta}$ is the propagation direction of the X-rays. In the framework of the Fresnel diffraction theory, the intensity detected at a distance $d$ after the sample is given by the squared modulus of the $2 \mathrm{D}$ convolution between the transmittance $T_{\theta}$ and the Fresnel propagator $P_{d}$ for a distance $d$ downstream of the object $[8,11,21]$ :

$$
I_{d, \theta}\left[\delta_{n}\right]=\left|T_{\theta}\left[\delta_{n}\right] * P_{d}\right|^{2},
$$

where

$$
P_{d}\left(X_{\theta}\right)=\frac{1}{i \lambda d} \exp \left(i \frac{\pi}{\lambda d}\left|X_{\theta}\right|^{2}\right) .
$$

The intensity $I_{d}$ operator can thus be considered as a nonlinear function of $\delta$.
2.2. Linear Approaches of the Phase Retrieval Problem. For each value of the projection $\theta$, the phase retrieval problem to recover the phase from the intensity patterns set in the $X_{\theta}=\left(x_{\theta}, z\right)$ plane has been linearized in the framework of several models. We first review some widely used relations between the phase and the measured intensity. The Fourier coordinates in the $X_{\theta}=\left(x_{\theta}, z\right)$ plane will be denoted as $\vec{k}=(\xi, \eta)$. For sufficiently small propagation distance $d$, the function $\varphi$ varies in an approximately linear way in $d$ and the phase can be written:

$$
\varphi\left(X_{\theta}\right)=\varphi_{d=0}\left(X_{\theta}\right)+\varphi_{1}\left(X_{\theta}\right) d
$$

(see [22-24]). In the small $d$ limit, the transport of intensity equation (TIE) leads to the linear approximating equation:

$$
g_{d}\left(X_{\theta}\right)=-\frac{d}{k} \Delta \varphi_{d=0}\left(X_{\theta}\right),
$$

where $\Delta$ is a Laplacian operator, $I_{d}$ is the intensity at a finite object-detector distance $d, g_{d}\left(X_{\theta}\right)=\left(I_{d}\left(X_{\theta}\right) / I_{d=0}\right)-1$ is the intensity contrast, $k=2 \pi / \lambda$ is the wave number of the incident monochromatic X-rays, and $\varphi_{d=0}$ is the phase shift in the exit plane of the object.

In the framework of the contrast transfer function (CTF) model $[14-18,25]$, in the small-relative-phase limit corresponding to the assumption,

$$
\left|\varphi_{d=0}\left(\vec{X}_{\theta}-\frac{\pi d \vec{k}}{k}\right)-\varphi_{d=0}\left(\vec{X}_{\theta}+\frac{\pi d \vec{k}}{k}\right)\right| \ll 1,
$$

the relation between the phase and the data $g_{d}$ can be written in the Fourier space with a linear operator $A$ :

$$
g_{d}=\mathscr{F}^{-1}\left(2 \sin \left(u\|\vec{k}\|^{2}\right) \mathscr{F}(\varphi)(\vec{k})\right)=A(\varphi),
$$

with $u=\pi d \lambda$ and where the 2-dimensional Fourier transform and its inverse are given by

$$
\begin{gathered}
\widehat{f}(\vec{k})=\mathscr{F} g(\vec{k})=\frac{1}{2 \pi} \int_{\vec{x} \in \mathbb{R}^{2}} e^{-i \vec{x} \cdot \vec{k}} f(\vec{x}) d \vec{x}, \\
\mathscr{F}^{-1} g(\vec{x})=\frac{1}{2 \pi} \int_{\vec{k} \in \mathbb{R}^{2}} e^{i \vec{x} \cdot \vec{k}} f(\vec{k}) d \vec{k} .
\end{gathered}
$$

This approach is more accurate than the TIE method which relies on a short propagation distance assumption. In the following, we consider only the singularity at the origin because the other singularities can be removed with a combination of several propagation distances.

\subsection{The Coupling of Phase Retrieval with the Radon Projection} Operator. The direct intensity operator can be rewritten with the Radon projection operator. We first summarize some properties of this projection operator [26]. Let $\Omega \subset \mathbb{R}^{2}$ be a bounded open domain, the mathematical model for $2 \mathrm{D}$ tomography is the Radon transform $R$ which maps a function $f \in L^{1}(\Omega)$ to its line integrals. 
Definition 1. Let $L\left(\theta, x_{\theta}\right)$ be the line defined by $L\left(\theta, x_{\theta}\right)=$ $\left\{y_{\theta} \bar{\theta}^{*}+x_{\theta} \bar{\theta}: y_{\theta} \in \mathbb{R}\right\}$, with $\bar{\theta}=(\cos (\theta), \sin (\theta))$ and $\bar{\theta}^{*}=$ $(-\sin (\theta), \cos (\theta))$, and let $R a$ be the range of the Radon transform and $Z=[0, \pi] \times R a$; the Radon transform for $f \in L^{1}(\Omega)$ is defined by

$$
R f\left(\theta, x_{\theta}\right)=R_{\theta} f\left(x_{\theta}\right)=\int_{t \in L\left(\theta, x_{\theta}\right) \cap \Omega} f(t) d t .
$$

Let $g \in L^{1}([0, \pi] \times \mathbb{R})$ and let $\bar{U}=(x, y)$; the adjoint $R^{*}: L^{2}(Z) \rightarrow L^{2}(\Omega)$ of $R$ is given by

$$
R^{*} g(\bar{U})=\int_{0}^{\pi} g(\theta, \bar{U} \cdot \bar{\theta}) d \theta
$$

The proof can be found in [26].

For parallel beam projection, with a beam parallel to the $X=(x, y)$ plane, $f \in L^{1}(\Sigma)$ and $L\left(\theta, x_{\theta}, z\right)$ the $L\left(\theta, x_{\theta}\right)$ line for the coordinate $z$ :

$$
I_{z} \otimes R f\left(\theta, x_{\theta}, z\right)=R_{\theta} f\left(x_{\theta}\right)=\int_{t \in L\left(\theta, x_{\theta}, z\right) \cap \Sigma} f(t) d t,
$$

where $\otimes$ denotes a tensor product and $I_{z}$ is the identity on the $z$ variable. The Sobolev spaces $H^{\alpha}$ are very useful to characterize the smoothing properties of the Radon transform. The Sobolev norms $\|\cdot\|_{H^{\alpha}}$ for functions in $\mathbb{R}^{n}$ are defined as

$$
\|f\|_{H^{\alpha}}^{2}=\int_{\mathbb{R}^{n}}\left(1+|\xi|^{2}\right)^{\alpha}|\mathscr{F}(f)(\xi)|^{2} d \xi .
$$

Let $g \in L^{1}([0, \pi] \times \mathbb{R})$; the one-dimensional Fourier $F_{p}(g)(\theta, \tau)$ is defined by $F_{p}(g)(\theta, \tau)=(1 / \sqrt{2 \pi}) \int_{\mathbb{R}} \exp ^{-i \tau p} g(\theta$, p)dp. Let $s \in \mathbb{R}$, the Sobolev space on $[0, \pi] \times \mathbb{R}$ denoted as $H^{0, s}([0, \pi] \times \mathbb{R})$, be the set of all distributions $g$ on $([0, \pi] \times \mathbb{R})$ such that the Fourier transform $F_{p}(g)$ is a function and such that the Sobolev norm of $g,\|g\|_{H^{0, s}(Z)}^{2}$, is finite [26]. The norm $\|g\|_{H^{0, s}(Z)}^{2}$ is defined as

$$
\|g\|_{H^{0, s}(Z)}=\int_{0}^{\pi} \int_{\mathbb{R}}\left(1+|\tau|^{2}\right)^{s}\left|F_{p}(g)(\theta, \tau)\right|^{2} d \tau d \theta .
$$

The Radon operator is a smoothing operator as detailed in the following proposition.

Proposition 2. Let $f$ be a distribution with a compact support; then, the following bound holds for $s \geq 0$ :

$$
\|R(f)\|_{H^{0, s+1 / 2}(Z)} \leq C\|f\|_{H^{s}\left(\mathbb{R}^{2}\right)} .
$$

The proof of this proposition can be found in [26]. It is now possible to use the mapping properties of the Radon transform in the context of phase contrast tomography and in a Hilbert spaces framework.

Proposition 3. Let $R a_{z}$ be the range of the Radon operator for the height $z$; the operator $\varphi=I_{z} \otimes R\left[\delta_{n}\right]: L^{2}(\Sigma) \rightarrow L^{2}([0, \pi] \times$ $\left.\Sigma_{z} \times \cup R a_{z}\right)$ is continuous.
Proof. For $s>0, H_{0}^{s}\left(\mathbb{R}^{2}\right)$ is continuously embedded in $L^{2}\left(\mathbb{R}^{2}\right)$ and thus for compactly supported functions, there is a positive constant $C_{1}$ such that

$$
\|f\|_{H^{s}\left(\mathbb{R}^{2}\right)} \leq C_{1}\|f\|_{L^{2}\left(\mathbb{R}^{2}\right)} .
$$

Using (18), there exists a positive constant $C$ such that

$$
\|R(f)\|_{H^{0,0}(Z)} \leq\|R(f)\|_{H^{0,1 / 2}(Z)} \leq C\|f\|_{L_{2}\left(\mathbb{R}^{2}\right)} .
$$

In a Hilbert spaces context, the Radon transform is thus a linear continuous operator from $L^{2}\left(\mathbb{R}^{2}\right)$ to $L^{2}(Z)$. For parallel beam projection, let $R a_{z}$ be the range of the Radon operator for the height $z$. For each value of $z$, the Radon operator is continuous from $L^{2}\left(\Sigma_{x} \times \Sigma_{y}\right)$ to $L^{2}\left([0, \pi] \times R a_{z}\right)$. By integration on the variable $z$, the operator $\varphi=I_{z} \otimes R\left[\delta_{n}\right]: L^{2}(\Sigma) \rightarrow$ $L^{2}\left([0, \pi] \times \Sigma_{z} \times \cup R a_{z}\right)$ is continuous.

With the Radon projection operator, (4) can be rewritten:

$$
\varphi_{\theta}\left[\delta_{n}\right]\left(X_{\theta}\right)=\frac{2 \pi}{\lambda} I_{z} \otimes R\left[1-\delta_{n}\right]\left(\theta, X_{\theta}\right) .
$$

\section{Regularization for Phase Retrieval}

In this section, we investigate the convergence properties of some regularization approaches for phase retrieval. In a generic way, we denote by $f$ the unknown phase to reconstruct, by $g$ the intensity data, and by $A$ the direct operator.

3.1. Preliminaries. Let us assume that the phase is defined on a bounded domain $\Omega$. It is well known that the Laplacian operator of the TIE approach $\Delta: L^{2}(\Omega) \rightarrow H^{-2}(\Omega)$ is continuous [27]. The phase operators involved in the direct problem can be understood as Fourier multiplication operators, with a Fourier multiplier $m(\vec{k})$ and written as

$$
\mathscr{F} A f(\vec{k})=m(\vec{k}) \mathscr{F} f(\vec{k}) .
$$

The Fourier multiplier of the CTF method is given by

$$
m(\vec{k})=\sin \left(\pi \lambda d\|\vec{k}\|^{2}\right)
$$

It is bounded and thus the direct operator in the CTF method is bounded from $L_{2}\left(\mathbb{R}^{2}\right)$ to $L_{2}\left(\mathbb{R}^{2}\right)$ with the Parseval theorem. Some functional properties are lost with the linearization of the direct forward problem. The analysis of the nonlinear direct operator shows that it is a compact operator [28] because of the convolution with the Fresnel propagator. These smoothing properties are lost in the linearized methods presented above. With the linearization, the Laplacian operator in the TIE version or the CTF direct operator is not compact operator.

The inverse Fourier multipliers $m(\vec{k})$ involved in the TIE or CTF approaches $m(\vec{k})=1 /\|\vec{k}\|^{2}$ and $m(\vec{k})=1 /$ $\sin \left(\pi \lambda d\|\vec{k}\|^{2}\right)$ define tempered distributions with singular kernels. It is necessary to use the finite part to give a sense to these distributions. 
Definition 4. Given a function $u \in L_{\text {loc }}^{1}\left(\mathbb{R}^{n}-\{0\}\right)$ and given a function $\phi \in C_{k}^{\infty}$, the finite part $f p$ of the integral $\int u(y) \phi(y) d y$ is defined as

$$
\begin{aligned}
f p \int u(y) \phi(y) d y= & \lim _{\epsilon \rightarrow 0} \int_{|y|>\epsilon} u(y)(\phi(y)-\phi(0)) d y \\
& +\int_{|y|>1} u(y) \phi(y) d y .
\end{aligned}
$$

The following proposition is useful to show that some finite part distributions are well defined.

Proposition 5. Let one assume that the function $u \in L_{\text {loc }}^{1}\left(\mathbb{R}^{n}-\right.$ $\{0\})$ satisfies the size condition

$$
|u(y)| \leq \frac{C}{|y|^{n}}, \quad y \neq 0 ;
$$

then, it is possible to define the finite part of $u$ [29-31].

Thus, $f \cdot p\left(1 /\|\vec{k}\|^{2}\right)$ and $f \cdot p\left(1 / \sin \left(\pi \lambda d\|\vec{k}\|^{2}\right)\right)$ are welldefined tempered distributions. For data $g \in L_{2}(\Omega)$, the product $m(\vec{k}) \times \widehat{g}$ defines a tempered distribution in $\mathcal{S}^{\prime}[29,30]$. The inverse Fourier transform of $m(\vec{k}) \times \widehat{g}$ is well defined. The data function $g$ has a bounded support, $g \in L_{2}(\Omega) \subset L_{1}(\Omega) \subset$ $L_{1}\left(\mathbb{R}^{2}\right)$, and the convolution of $m$ and $g$ is well defined [2931].

Distribution solutions are obtained because of the singularity of the Fourier multiplier at the origin. The inverse problem formulated with CTF or TIE approach is not a classic linear problem with a compact operator $A: X \rightarrow Y$ between Hilbert spaces $X$ and $Y$. The inverse is defined in a distributional sense. A regularized distribution is obtained when the zero frequency component of the test function is suppressed. It is the idea of the "quasiparticule" method detailed below. More generally, it is possible to construct an approximate inverse $\widetilde{A}_{\gamma}: L_{2}\left(\mathbb{R}^{2}\right) \rightarrow L_{2}(\Omega)$ with a bounded kernel in $L_{2}$. Let $\widetilde{A}_{\gamma}$ be the approximate inverse operator, depending on the regularization parameter $\gamma$. This approximate inverse must verify the pointwise convergence, for all $f \in L_{2}(\Omega)$ :

$$
\lim _{\gamma \rightarrow 0} \widetilde{A}_{\gamma} A f=f
$$

Let $f^{\gamma, \delta}$ be the solution obtained from the noisy data $g^{\delta}$, $f^{\gamma, \delta}=\widetilde{A}_{\gamma} g^{\delta}$. We assume that the noisy data $g^{\delta}$ and the unnoisy data $g$ are such that

$$
\left\|g-g^{\delta}\right\| \leq \delta
$$

where $\delta$ is the noise level and $\|\cdot\|$ is the $L_{2}$ norm. We are interested in this work in the convergence of various regularization methods. We will make use of the following inequality for $f \in L_{2}\left(\mathbb{R}^{2}\right)$ :

$$
\left\|f-f^{\alpha, \delta}\right\| \leq \delta\left\|\widetilde{A}_{\gamma}\right\|+\left\|\widetilde{A}_{\gamma} A f-f\right\| .
$$

3.2. Regularization for CTF. In this section, we investigate the convergence properties of some regularization methods for CTF. Several methods of regularization have been tested [14-18] to solve this linear inverse problem, like the quadratic Tikhonov regularization used for the classical inverse problems or the "quasiparticule approach." We detail here the convergence properties for the Tikhonov and quasiparticule approach and for the approximate inverse obtained with mollification.

3.2.1. Tikhonov Regularization for CTF. The Tikhonov regularization method is a convergent regularization method.

Proposition 6. The Tikhonov regularization method is a convergent regularization method with an optimal convergence rate $\left\|f-f^{\alpha, \delta}\right\| \sim \delta^{1 / 3}$.

Proof. For the Tikhonov regularization, the approximate inverse can be written:

$$
\widetilde{A}_{\gamma}=\left(\gamma I+A^{*} A\right)^{-1} A^{*},
$$

where $A$ is a Fourier multiplication operator given in (10). The approximate inverse for CTF is also a Fourier multiplication operator:

$$
\widetilde{A}_{\gamma} f=\mathscr{F}^{-1}(m(\vec{k}) \mathscr{F} f(\vec{k})) .
$$

With $u=\pi \lambda D$, the Fourier multiplier $m(\vec{k})$ is given by

$$
m(\vec{k})=\frac{\sin \left(u\|\vec{k}\|^{2}\right)}{\gamma+\sin \left(u\|\vec{k}\|^{2}\right)^{2}} .
$$

We first obtain an upper bound for the norm of the operator $\widetilde{A}_{\gamma}$ :

$$
\begin{aligned}
\left\|\widetilde{A}_{\gamma} f\right\| & =\left\|\mathscr{F}^{-1} \frac{\sin \left(u\|\vec{k}\|^{2}\right)}{\gamma+\sin ^{2}\left(u\|\vec{k}\|^{2}\right)} \mathscr{F} f\right\| \\
& =\left\|\frac{\sin \left(u\|\vec{k}\|^{2}\right)}{\gamma+\sin ^{2}\left(u\|\vec{k}\|^{2}\right)} \mathscr{F} f\right\| \\
& \leq \sup _{\vec{k}} \frac{\left|\sin \left(u\|\vec{k}\|^{2}\right)\right|}{\gamma+\sin ^{2}\left(u\|\vec{k}\|^{2}\right)}\|f\|,
\end{aligned}
$$

where the first equality results from Plancherel's theorem.

With the identity

$$
\frac{x}{\gamma+x^{2}} \leq \frac{1}{2 \sqrt{\gamma}},
$$

we obtain

$$
\left\|\widetilde{A}_{\gamma}\right\| \leq \frac{C_{1}}{\sqrt{\gamma}} .
$$


For $f \in L_{2}(\Omega)$, we have

$$
\left(I-\widetilde{A}_{\gamma} A\right) f=\mathscr{F}^{-1} \frac{\gamma}{\gamma+\left(\sin \left(u\|\vec{k}\|^{2}\right)\right)^{2}} \mathscr{F} f(\vec{k}) .
$$

With the change of variable, $\vec{k}=\vec{k}^{\prime} \gamma^{1 / 4}$ and with the identity $\sin (x) \geq 2 x / \pi$, for $x \in[0, \pi / 2]$, we obtain

$$
\begin{aligned}
& \left\|\left(I-\widetilde{A}_{\gamma} A\right) f\right\|^{2} \\
& =\left\|\frac{\gamma}{\gamma+\left(\sin \left(u\|\vec{k}\|^{2}\right)\right)^{2}} \mathscr{F} f(\vec{k})\right\|^{2} \\
& \leq\left\|\frac{\gamma}{\gamma+\left((2 / \pi) u\|\vec{k}\|^{2}\right)^{2}} \mathscr{F} f(\vec{k})\right\|^{2} \\
& \leq \int\left|\frac{1}{1+\left((2 / \pi) u\left\|\vec{k}^{\prime}\right\|^{2}\right)^{2}} \mathscr{F} f\left(\vec{k}^{\prime \gamma^{1 / 4}}\right)\right|^{2} \gamma^{1 / 2} d \vec{k}^{\prime} .
\end{aligned}
$$

From the Rieman-Lebesgue convergence theorem, the Fourier transform is a continuous function. Thus, there is a constant positive $C_{2}$ such that

$$
\left\|\left(I-\widetilde{A}_{\gamma} A\right) f\right\| \leq C_{2} \gamma^{1 / 4}
$$

Reporting in (27), we obtain for the Tikhonov regularization

$$
\left\|f-f^{\alpha, \delta}\right\| \leq \delta \frac{C_{1}}{\sqrt{\gamma}}+C_{2} \gamma^{1 / 4}
$$

and thus the optimal convergence rate is obtained for the a priori choice, $\gamma \sim \delta^{4 / 3}$, and the convergence rate is

$$
\left\|f-f^{\alpha, \delta}\right\| \sim \delta^{1 / 3}
$$

3.2.2. Quasiparticle Regularization. In the framework of the quasiparticule approach [22-24], the intensity contrast is filtered in the Fourier space $\mathscr{F}\left(g_{z}\right)(\vec{k})$ and is replaced by $\mathscr{F}_{\Theta}\left(g_{z}\right)(\vec{k})$ :

$$
\mathscr{F}_{\Theta}\left(g_{z}\right)(\vec{k})=\Theta\left(\left|\sin \left(\pi \lambda d\|\vec{k}\|^{2}\right)\right|-\epsilon\right) \mathscr{F}\left(g_{z}\right)(\vec{k}),
$$

where $\Theta$ denotes the Heaviside step function and $\epsilon$ is a threshold for this filter.

Considering only the singularity of the multiplier at the origin, this threshold $\epsilon$ determines a threshold $\gamma>0$ such that

$$
\sin \left(\pi \lambda d \gamma^{2}\right)=\epsilon
$$

Let $H_{\gamma}(\vec{k})$ be the filter function defined by

$$
H_{\gamma}(\vec{k})= \begin{cases}0, & \text { if }\|\vec{k}\| \leq \gamma, \\ 1, & \text { if }\|\vec{k}\|>\gamma .\end{cases}
$$

The regularized inverse can be written:

$$
\begin{gathered}
\widetilde{A}_{\gamma} f=\mathscr{F}^{-1} \frac{H_{\gamma}(\vec{k})}{\sin \left(u\|\vec{k}\|^{2}\right)} \mathscr{F} f(\vec{k}), \\
\left\|\widetilde{A}_{\gamma} f\right\| \leq\left\|\mathscr{F}^{-1} \frac{H_{\gamma}(\vec{k})}{\sin \left(u\|\vec{k}\|^{2}\right)} \mathscr{F} f(\vec{k})\right\| \\
\leq \frac{1}{\sin \left(u \gamma^{2}\right)}\left\|H_{\gamma}(\vec{k}) \mathscr{F} f(\vec{k})\right\| \\
\leq \frac{1}{\sin \left(u \gamma^{2}\right)}\|\mathscr{F} f(\vec{k})\|=\frac{1}{\sin \left(u \gamma^{2}\right)}\|f\| .
\end{gathered}
$$

With the identity $\sin (x) \geq 2 x / \pi$, for $x \in[0, \pi / 2]$, we obtain that there is a constant $C$ such that

$$
\left\|\widetilde{A}_{\gamma}\right\| \leq \frac{C}{\gamma^{2}} \text {. }
$$

Moreover, we have

$$
\begin{gathered}
\left(I-\widetilde{A}_{\gamma} A\right) f=\mathscr{F}^{-1}\left(1-H_{\gamma}(\vec{k})\right) \mathscr{F} f, \\
\left\|\left(I-\widetilde{A}_{\gamma} A\right) f\right\|=\left\|1_{\|\vec{k}\| \leq \gamma} \mathscr{F} f\right\| .
\end{gathered}
$$

By the Lebesgue dominated convergence theorem, this term tends to zero as $\gamma \rightarrow 0$. Yet it is impossible to derive any convergence rate of the method as $\gamma \rightarrow 0$ without any assumption on the position of the spectra of $f$ with respect to the threshold. In the more favorable case, if the spectra of the function $f$ are above $\gamma$, this term is zero and

$$
\left\|x-x^{\alpha, \delta}\right\| \leq \delta \frac{C}{\gamma^{2}} .
$$

The convergence is obtained for a choice of the regularization parameter such that $\delta / \gamma^{2} \rightarrow 0$ as $\delta \rightarrow 0$.

3.2.3. Regularization with Mollification. It is not possible to derive any convergence rate for the "quasiparticule" regularization without any assumption about the spectrum of the function to regularize. In this section, we detail the regularization with mollification which is valid without any restrictive assumption.

We consider a mollifier $\phi \in L^{1}\left(\mathbb{R}^{2}\right)$ such that

$$
\mathscr{F} \phi(0)=\int_{\mathbb{R}^{2}} \phi(x) d x=1,
$$

and we assume that there exists $s>0$ such that

$$
|1-\mathscr{F} \phi(\vec{k})| \sim\|\vec{k}\|^{s} \quad \text { as } \vec{k} \rightarrow 0 .
$$


Typical examples of mollifier are the Gaussian function

$$
\phi(x)=(2 \pi)^{-1} \exp \left(-\frac{\|x\|^{2}}{2}\right)
$$

or

$$
\phi^{\nu}(x)=A \begin{cases}\left(1-\|x\|^{2}\right)^{\nu}, & \text { if }\|x\| \leq 1 \\ 0, & \text { if }\|x\|>1\end{cases}
$$

where $A$ is a normalizing constant.

We consider as approximate inverse the operator defined for $f \in L^{2}(\Omega)$ by

$$
\widetilde{A}_{\gamma} f=\mathscr{F}^{-1} \frac{1-\mathscr{F} \phi(\vec{k} / \gamma)}{\left(\sin \left(u\|\vec{k}\|^{2}\right)\right)} \mathscr{F} f(k) .
$$

Proposition 7. The CTF formulation of the phase retrieval inverse problem can be regularized by the mollification regularization with an exponent $s=2$. The optimal convergence rate is $\left\|f-f^{\alpha, \delta}\right\| \sim \delta^{1 / 2}$.

Proof. Let us assume $s=2$; then,

$$
\begin{aligned}
\left\|\widetilde{A}_{\gamma} f\right\| & =\left\|\mathscr{F}^{-1} \frac{1-\mathscr{F} \phi(\vec{k} / \gamma)}{\left(\sin \left(u\|\vec{k}\|^{2}\right)\right)} \mathscr{F} f(k)\right\| \\
& =\left\|\frac{1-\mathscr{F} \phi(\vec{k} / \gamma)}{\left(\sin \left(u\|\vec{k}\|^{2}\right)\right)} \mathscr{F} f(k)\right\| \\
& \leq \sup _{k} \frac{|1-\mathscr{F} \phi(\vec{k} / \gamma)|}{\left|\sin \left(u\|\vec{k}\|^{2}\right)\right|}\|\mathscr{F} f(\vec{k})\| \\
& =\sup _{k} \frac{|1-\mathscr{F} \phi(\vec{k} / \gamma)|}{\left|\sin \left(u\|\vec{k}\|^{2}\right)\right|}\|f\| .
\end{aligned}
$$

From the Rieman-Lebesque theorem, $\mathscr{F} \phi$ is continuous and decreases at infinity and thus $|1-\mathscr{F} \phi(\vec{k} / \gamma)| /\left|\sin \left(u\|\vec{k}\|^{2}\right)\right|$ is bounded for the higher value of $\vec{k}$.

As $\vec{k} \rightarrow 0$,

$$
\frac{|1-\mathscr{F} \phi(\vec{k} / \gamma)|}{\left|\sin \left(u\|\vec{k}\|^{2}\right)\right|} \sim \frac{\gamma^{-s}\|\vec{k}\|^{s}}{u\|\vec{k}\|^{2}}
$$

for $s=2$, there is a constant $C_{1}$ such that

$$
\frac{|1-\mathscr{F} \phi(\vec{k} / \gamma)|}{\left|\sin \left(u\|\vec{k}\|^{2}\right)\right|} \leq C_{1} \gamma^{-2}
$$

Thus, we obtain

$$
\left\|\widetilde{A}_{\gamma}\right\| \leq C_{1} \gamma^{-2}
$$

with the definition of approximate inverse

$$
\left(I-\widetilde{A}_{\gamma} A\right) f=\mathscr{F}^{-1}\left(\mathscr{F} \phi\left(\frac{\vec{k}}{\gamma}\right) \mathscr{F} f(\vec{k})\right) .
$$

The mollifier is defined on $\mathbb{R}^{2}$ and thus

$$
\mathscr{F} \phi\left(\frac{\vec{k}}{\gamma}\right)=\gamma^{2} \mathscr{F}(\phi(x \gamma)) ;
$$

we obtain

$$
\begin{aligned}
\left\|\left(I-\widetilde{A}_{\gamma} A\right) f\right\| & =\gamma^{2}\|\mathscr{F}(\phi(x \gamma)) \mathscr{F} f(k)\| \\
& \leq \gamma^{2}\|\mathscr{F} f(\vec{k})\| \\
& \leq \gamma^{2}\|f\| .
\end{aligned}
$$

With (27),

$$
\left\|f-f^{\alpha, \delta}\right\| \leq \delta C_{1} \gamma^{-2}+\gamma^{2}\|f\| ;
$$

the optimal convergence rate obtained with the choice $\gamma \sim$ $\delta^{1 / 4}$ is

$$
\left\|f-f^{\alpha, \delta}\right\| \sim \delta^{1 / 2}
$$

\section{Regularization for Phase Contrast Tomography}

In this section, we present some regularization methods for phase contrast tomography which are combination of regularization methods for tomography and phase retrieval. We will use the mollifier regularization for the phase retrieval problem (Section 3.2.3).

4.1. Inversion Formula for Tomography and Regularized Inversion Approaches. We first summarize the well-known filtered backprojection inversion formula for a $2 \mathrm{D}$ geometry.

Definition 8. The Riesz potential $\Lambda^{\alpha}: H^{t}\left(\mathbb{R}^{d}\right) \rightarrow H^{t-\alpha}\left(\mathbb{R}^{d}\right)$ for $\alpha>-d$ is defined by

$$
\mathscr{F}\left(\Lambda^{\alpha} f(\vec{k})\right)=\|\xi\|^{\alpha} \mathscr{F} f(\vec{k}) .
$$

The Riesz potential is an isomorphism of Hilbert spaces. For $\alpha<0$, it is a compact operator. The Riesz potential used for tomography reconstruction, $\Lambda_{s}$, for $g \in \mathscr{C}_{c}^{\infty}([0, \pi] \times \mathbb{R})$, is the operator with Fourier multiplier $|\xi|$ :

$$
\Lambda_{s}(g)=\mathscr{F}_{s}^{-1}\left(|\xi| \mathscr{F}_{s} g\right),
$$

where the partial Fourier transform of $g \in \mathscr{C}_{c}^{\infty}([0, \pi] \times \mathbb{R})$ in the $s$ variable is defined by the formula

$$
\mathscr{F}_{s} g(\phi, \xi)=\frac{1}{\sqrt{2 \pi}} \int_{-\infty}^{\infty} e^{-i s \xi} g(\phi, s) d s .
$$

The inversion formula is summarized by the following theorem [26]. 
Theorem 9. Let $f \in \mathscr{C}_{c}^{\infty}\left(\mathbb{R}^{2}\right)$, the set of infinitely differentiable functions with $2 \mathrm{D}$ compact support; then, $f$ can be reconstructed with the formula

$$
f=\frac{1}{4 \pi} R^{*} \Lambda_{s} R f
$$

where $R^{*}$ is the adjoint of the Radon transform.

This reconstruction formula can be generalized for parallel beam projection with tensor product of identity operators on the $z$ coordinate. In this case, $\xi$ is the spatial frequency associated to the $x_{\theta}$ axis (Figure 1). For parallel beam projection, the reconstruction formula is

$$
f(x)=\frac{1}{4 \pi}\left(I_{z} \otimes R^{*}\right)\left(I_{\theta} \otimes I_{z} \otimes \Lambda_{s}\right)\left(I_{z} \otimes R\right) f(x) .
$$

Several regularized inverse operators have been studied in the literature, based on truncated filtered backprojection [26, 32 ] or on the approximate inverse obtained with mollifiers [33].

In the framework of the truncated filtered backprojection, the approximate inversion formula can be written as $f=$ $(1 / 4 \pi) R^{*}(\Pi * R f)$, where $\Pi$ is the inverse Fourier transform of the truncated multiplier $|\xi|$ and $*$ denotes the convolution in the $s$ variable:

$$
\mathscr{F}_{s} \Pi(\xi)= \begin{cases}|\xi|, & \text { if }|\xi| \leq \omega \\ 0, & \text { if }|\xi|>\omega\end{cases}
$$

where $\omega$ is a cutoff frequency. In the following, we denote by $\Lambda_{\gamma}$ the regularized Riesz potential obtained with this kernel, with the power law $\omega=\gamma^{-\tau}$, where $\tau$ is a positive constant.

In the framework of the approximate inverse theory obtained with mollifiers, the approximate inverse is given by

$$
f * e=R^{*}\left(v *{ }_{s} R f\right),
$$

where $v$ is the reconstruction kernel and $e$ is a mollifier approximating the $\delta$ distribution, centered about 0 and with mean value 1 . The reconstruction kernel and the mollifier are related by

$$
v=\Lambda R e .
$$

A family of reconstruction kernel is obtained for $\gamma>0$ as

$$
\begin{aligned}
& e_{\gamma}(x)=\gamma^{-2} e\left(\frac{x}{\gamma}\right), \\
& v_{\gamma}(x)=\gamma^{-2} v\left(\frac{x}{\gamma}\right) .
\end{aligned}
$$

The theory of the approximate inverse Hilbert space is fully presented in $[33,34]$. This method of reconstruction allows for error estimates. Given measured data $g^{\delta}$, assuming that the range of the Radon transform is $[-1,1]$, the reconstruction formula can be written, for $y \in \Omega$ :

$$
\begin{aligned}
\widetilde{R}_{\gamma} g^{\delta}(y) & =f(y) \\
& =\gamma^{-2} \int_{0}^{\pi} \int_{-1}^{1} g^{\delta}(\theta, s) v\left(\theta, \frac{s-\langle y, \bar{\theta}\rangle}{\gamma}\right) d s d \theta .
\end{aligned}
$$

For a 3D geometry, an approximate inverse of the Radon projector can be obtained with the tensorial product $\widetilde{R}_{\gamma} \otimes I_{z}$.

4.2. The Approximate Inverse for Phase Contrast Tomography. The phase contrast tomography problem can be written in a generic way as

$$
U(f)=\left(A \otimes I_{\theta}\right)\left(I_{z} \otimes R\right) f=g
$$

where $f$ denotes in this section the unknown imaginary index part $\delta_{n}$ and $g$ is the intensity data. The operator $I_{z} \otimes R$, $\varphi=I_{z} \otimes R\left[\delta_{n}\right]: L^{2}(\Sigma) \rightarrow L^{2}\left([0, \pi] \times \Sigma_{z} \times \cup R a_{z}\right)$, is linear and continuous. The same result is true for the operator $A \otimes I_{\theta}: L^{2}\left([0, \pi] \times \Sigma_{z} \times \cup R a_{z}\right) \rightarrow L^{2}\left([0, \pi] \times \mathbb{R}^{2}\right)$.

The approximate inverse of the direct operator can be expressed as

$$
\widetilde{U}=\left(\widetilde{R}_{\gamma} \otimes I_{z}\right)\left(\widetilde{A}_{\gamma} \otimes I_{\theta}\right)
$$

where $\widetilde{A}_{\gamma}$ and $\widetilde{R}_{\gamma}$ are approximate inverses for the Radon projection operator and the phase retrieval operator $A$. We will study successively the convergence of two inversion schemes: a coupling of the CTF approach regularized with mollifiers with the tomography inverse obtained with truncated filtered backprojection or with mollifiers.

4.2.1. Coupling of CTF and Riesz Potential Regularized with a Rectangular Window. We first study the case of a coupling of CTF regularized with mollifiers with a Riesz potential regularized with a rectangular window. In this case, the approximate inverse $\widetilde{U}$ is given by

$$
\begin{aligned}
\widetilde{U}= & \left(I_{z} \otimes R^{*}\right)\left(I_{\theta} \otimes I_{z} \otimes \Lambda_{\gamma}\right)\left(I_{\theta} \otimes \widetilde{A}\right) \\
= & \left(I_{z} \otimes R^{*}\right)\left(I_{\theta} \otimes I_{z} \otimes \mathscr{F}_{1}^{-1}|\xi| 1_{|\xi| \leq \omega} \mathscr{F}_{1}\right) \\
& \cdot\left(I_{\theta} \otimes \mathscr{F}_{2}^{-1} m(\xi, \eta) \mathscr{F}_{2}\right) .
\end{aligned}
$$

In this formula, $m(\xi, \eta)$ is the Fourier multiplier of the CTF approach regularized with the mollifier approach and $\xi$ and $\eta$ are the Fourier coordinates associated with the $x_{\theta}$ and $z$ axes. We obtain a formula similar to the one in Gureyev et al.s paper [25]. We demonstrate in the following that the inversion obtained with this method is convergent.

Proposition 10. Let us assume that, for each $z, f(\cdot, \cdot, z) \in$ $H^{0,1 / 2+\epsilon}\left(\mathbb{R}^{2}\right)$. The coupling of mollifier regularization for CTF with an inversion method for tomography based on a Riesz potential regularized with a rectangular window is a convergent regularization method. The method is convergent for $\tau<$ 4/3. The optimal convergence rate is obtained as $\left\|f-f^{\alpha, \delta}\right\| \sim$ $\delta^{\tau \epsilon /(\tau \epsilon+\tau+2)}$ for $\tau \epsilon<2-\tau$ and $\left\|f-f^{\alpha, \delta}\right\| \sim \delta^{(2-\tau) / 4}$ for $\tau \epsilon>2-\tau$.

Proof. In order to use a single regularization parameter, we assume that the threshold for the Riesz operator is given by 
$\omega=\gamma^{-\tau}$, where $\tau$ is a positive constant. We first obtain a bound for the norm of the truncated Riesz potential:

$$
\begin{aligned}
\left\|\Lambda_{\gamma} f\right\| & =\left\|\mathscr{F}^{-1} 1_{|\xi| \leq \omega}|\xi| \mathscr{F} f\right\| \\
& =\left\|1_{|\xi| \leq \omega}|\xi| \mathscr{F} f\right\| \\
& \leq \omega\|\mathscr{F} f\|=\gamma^{-\tau}\|f\| .
\end{aligned}
$$

The norm of a tensorial product of operators is the product of the norm of the operators. With the upper bound for the norm of the approximate inverse obtained with the mollifier regularization for the CTF operator (54) and the fact that the operator $R^{*}$ is bounded, we get

$$
\|\widetilde{U}\| \leq C_{1} \gamma^{-\tau-2}
$$

Ignoring tensorial products with identity operators for the sake of simplicity, we have the equality

$$
\begin{aligned}
I-\widetilde{U} U & =I-R^{*} \Lambda_{\gamma}(\widetilde{A} A-I) R+R^{*} \Lambda R+R^{*}\left(\Lambda_{\gamma}-\Lambda\right) R \\
& =-R^{*} \Lambda_{\gamma}(\widetilde{A} A-I) R+R^{*}\left(\Lambda_{\gamma}-\Lambda\right) R ;
\end{aligned}
$$

we obtain

$$
\|(I-\widetilde{U} U) f\| \leq\left\|R^{*} \Lambda_{\gamma}(\widetilde{A} A-I) R f\right\|+\left\|R^{*}\left(\Lambda_{\gamma}-\Lambda\right) R f\right\| .
$$

Let us assume that $f \in H^{1 / 2+\epsilon}\left(\mathbb{R}^{2}\right)$, with a constant $\epsilon>$ 0 ; then, $g=R f \in H^{0,1+\epsilon}(Z)$, and $|\xi|^{1+\epsilon} \mathscr{F} g \in L^{2}$ (Proposition 2):

$$
\begin{aligned}
\left\|\left(\Lambda_{\gamma}-\Lambda\right) R f\right\| & =\left\|1_{|\xi| \geq \omega}|\xi| \mathscr{F} R f\right\| \\
& =\left\|1_{|\xi| \geq \omega}|\xi|^{1+\epsilon}|\xi|^{-\epsilon} \mathscr{F} R f\right\| \\
& \leq\left\|1_{|\xi| \geq \omega}|\xi|^{1+\epsilon}|\xi|^{-\epsilon} \mathscr{F} R f\right\| \\
& \leq \sup _{|\xi| \geq \omega}|\xi|^{-\epsilon}\left\||\xi|^{1+\epsilon} \mathscr{F} R f\right\| \\
& \leq \gamma^{\tau \epsilon}\left\||\xi|^{1+\epsilon} \mathscr{F} R f\right\| \\
& \leq C_{2} \gamma^{\tau \epsilon},
\end{aligned}
$$

where $C_{2}$ is a positive constant. The same bound can be obtained for $3 \mathrm{D}$ geometry with parallel beam projection. With the bound obtained for $(\widetilde{A} A-I) f$ for mollifier regularization, we have

$$
\begin{aligned}
\|(I-\widetilde{U} U) f\| & \leq\left\|R^{*} \Lambda_{r}(\widetilde{A} A-I) R f\right\|+\left\|R^{*}\left(\Lambda_{r}-\Lambda\right) R f\right\| \\
& \leq C_{2} \max \left(\gamma^{2-\tau}, \gamma^{\tau \epsilon}\right) \\
& \leq C_{2} \gamma^{\min (2-\tau, \tau \epsilon)} .
\end{aligned}
$$

We thus obtain the following bound:

$$
\left\|f-f^{\alpha, \delta}\right\| \leq C_{1} \delta \gamma^{-\tau-2}+C_{2} \gamma^{\min (2-\tau, \tau \epsilon)} .
$$

The method is convergent for $\tau<2$. The optimal convergence rate is obtained as $\left\|f-f^{\alpha, \delta}\right\| \sim \delta^{\tau \epsilon /(\tau \epsilon+\tau+2)}$ for $\tau \epsilon<2-\tau$ and the choice $\gamma=\delta^{1 /(\tau \epsilon+\tau+2)}$ and $\left\|f-f^{\alpha, \delta}\right\| \sim \delta^{(2-\tau) / 4}$ for $\tau \epsilon>2-\tau$ and the choice $\gamma=\delta^{1 / 4}$.

4.2.2. Coupling of CTF and Mollified Approximate Inverse. In this section, we study the coupling of mollifier regularization for CTF with the inverse Radon transform obtained with the approximate inverse method based on mollifier kernels [33]. Classical results for mollifier show that $e * f$ converge to $f$ in $L^{2}$ as $\gamma \rightarrow 0$. To have a more precise bound, we will use the following lemma about difference quotients in Sobolev spaces [27].

Definition 11. Given a unit vector $v$ and a measurable function $u$, the $h$-translate of $u$ in the direction $v$ is denoted as $\tau_{v}^{h} u$ and defined by

$$
\tau_{v}^{h} u(x)=u(x+v h) .
$$

For a measurable function $u$, the difference quotient of $u$ in the coordinate direction $e_{i}$ of length $h \neq 0$ is the measurable function defined by

$$
\Delta_{i}^{h} u=\frac{\tau_{e_{i}}^{h} u-u}{h} .
$$

Proposition 12. Let $u \in W^{1, p}\left(\Omega^{\prime}\right)$ and let $D u$ be the weak derivative of $u$. Then, $\Delta^{h} u \in L^{p}(\Omega)$ for any $\Omega \subset \Omega^{\prime}$, with $h<$ $\operatorname{dist}\left(\Omega, \partial \Omega^{\prime}\right)$ and

$$
\left\|\Delta^{h} u\right\|_{p ; \Omega} \leq\|D u\|_{p ; \Omega^{\prime}}
$$

The convergence results are summarized by the following proposition.

Proposition 13. Let us assume that $f$ can be extended to a larger domain $\Omega^{\prime}$ such that $f \in W^{1,2}\left(\Omega^{\prime}\right)$. The coupling of the mollifier regularization for CTF and of the regularization of the inverse Radon transform with the approximate inverse approach based on mollifiers is a convergent method with a convergence rate $\left\|f-f^{\alpha, \delta}\right\| \sim \delta^{1 / 8}$. 
Proof. We first bound the norm of $\widetilde{R}_{\gamma}$. We assume that the range of the Radon transform is $V=[-1,1]$, with the Young and Schwartz inequalities we obtain, for $y \in \Omega$ :

$$
\begin{aligned}
& \left|\widetilde{R}_{\gamma} g(y)\right|^{2} \\
& \quad=\left(\gamma^{-2} \int_{0}^{\pi} \int_{-1}^{1}\left|g(\theta, s) v\left(\theta, \frac{s-\langle y, \bar{\theta}\rangle}{\gamma}\right)\right| d s d \theta\right)^{2} \\
& \quad \leq\left(\int_{0}^{\pi}\|g(\theta, s)\|_{L_{2}(V)}\left\|v_{\gamma}(\theta, s)\right\|_{L_{2}(V)} d \theta\right)^{2} \\
& \quad \leq\left(\int_{0}^{\pi}\|g(\theta, s)\|_{L_{2}(V)}^{2} d \theta\right)\left(\int_{0}^{\pi}\left\|v_{\gamma}(\theta, s)\right\|_{L_{2}(V)}^{2} d \theta\right) .
\end{aligned}
$$

The Fourier slice theorem can be written [32]:

$$
\frac{1}{\sqrt{2 \pi}} \mathscr{F}_{s} R f(\theta, \sigma)=\mathscr{F}(f)(\sigma \bar{\theta})=\widehat{f}(\sigma \bar{\theta})
$$

where $\mathscr{F}_{s}$ denotes the Fourier transform with respect to the $s$ variable and $\mathscr{F} f=\widehat{f}$ is the $2 \mathrm{D}$ Fourier transform; thus,

$$
\begin{aligned}
\Lambda_{s} R_{\theta} e_{\gamma}(s) & =\sqrt{2 \pi} \mathscr{F}_{s}^{-1}|\sigma| \widehat{e}_{\gamma}(\sigma \bar{\theta}), \\
\left\|\Lambda_{s} R_{\theta} e_{\gamma}\right\|_{L_{2}(V)}^{2} & =2 \pi\left\||\sigma| \widehat{e}_{\gamma}(\sigma \bar{\theta})\right\|_{L_{2}(V)}^{2} \\
& =2 \pi \int|\sigma|^{2}\left|\widehat{e}_{\gamma}(\sigma \bar{\theta})\right|^{2} d \sigma \\
& =2 \pi \int|\sigma|^{2}|\hat{e}(\gamma \sigma \bar{\theta})|^{2} d \sigma \\
& =2 \pi \gamma^{-3} \int|\sigma|^{2}|\hat{e}(\sigma \bar{\theta})|^{2} d \sigma \\
\int_{0}^{\pi}\left\|\Lambda_{s} R_{\theta} e_{\gamma}\right\|_{L_{2}(V)}^{2} d \theta & \\
= & \gamma^{-3} \int_{0}^{\pi} 2 \pi \int|\sigma|^{2}|\hat{e}(\sigma \bar{\theta})|^{2} d \sigma d \theta \\
= & \gamma^{-3} \int_{\mathbb{R}^{2}}\|\vec{k}\||\hat{e}(\vec{k})|^{2} d \vec{k} \\
\leq & \gamma^{-3} \int_{\mathbb{R}^{2}}\left(1+\|\vec{k}\|^{2}\right)^{\frac{1}{2}}|\hat{e}(\vec{k})|^{2} d \vec{k} \\
= & \gamma^{-3}\|e\|_{1 / 2},
\end{aligned}
$$

with a polar change of variable. By integration on the variable $y$, there is a positive constant $C$ such that

$$
\left\|\widetilde{R}_{\gamma}\right\| \leq C \gamma^{-3 / 2}
$$

We first bound the norm $\left\|e_{\gamma} * f-f\right\|$ :

$$
\begin{aligned}
e_{\gamma} & * f(x)-f(x) \\
& =\int e_{\gamma}(y)(f(x-y)-f(x)) d y \\
& =\gamma^{-2} \int e\left(\frac{y}{\gamma}\right)(f(x-y)-f(x)) d y \\
& =\int e(z)(f(x-\gamma z)-f(x)) d z .
\end{aligned}
$$

With the Hölder inequality, we obtain

$$
\begin{aligned}
& \left|e_{\gamma} * f(x)-f(x)\right| \\
& \leq\left(\int e(z)\left(|f(x-\gamma z)-f(x)|^{2}\right) d z\right)^{1 / 2}\left(\int e(z) d z\right)^{1 / 2} \\
& \leq\left(\int e(z)\left(|f(x-\gamma z)-f(x)|^{2}\right) d z\right)^{1 / 2} .
\end{aligned}
$$

By integration,

$$
\left\|e_{\gamma} * f-f\right\|^{2} \leq \int e(z)\|f(x-\gamma z)-f(x)\|^{2} d z .
$$

Let us note $d(\Omega)$, the diameter of the domain $\Omega$.

Let us assume that $f$ can be extended to a larger bounded domain $\Omega^{\prime}$, such that $f \in W^{1, p}\left(\Omega^{\prime}\right)$ with $\gamma d(\Omega)<\operatorname{dist}(\Omega$, $\partial \Omega^{\prime}$ ). Then, with proposition (8) with $p=2$, we obtain

$$
\left\|e_{\gamma} * f-f\right\| \leq C_{2} \gamma,
$$

for a positive constant $C_{2}$.

The regularized inverse can be written as $\widetilde{U}=\left(I_{z} \otimes \widetilde{R}_{\gamma}\right)\left(I_{\theta} \otimes\right.$ $\left.\widetilde{A}_{\gamma}\right)$. Using (86) and (54) for the mollifiers regularization of CTF, we thus obtain the bound

$$
\|\widetilde{U}\| \leq C_{1} \gamma^{-7 / 2},
$$

for a positive constant $C_{1}$. From the identity

$$
I-\widetilde{U} U=I-\widetilde{R}_{\gamma} R-R_{\gamma}\left(A_{\gamma} A-I\right) R,
$$

and with (90) and (57), we obtain for $C_{2} \geq 0$

$$
\|(I-\widetilde{U} U) f\| \leq C_{2} \max \left(\gamma, \gamma^{1 / 2}\right) \leq C_{2} \gamma^{1 / 2} .
$$

With (27), we obtain

$$
\left\|f-f^{\alpha, \delta}\right\| \leq C_{1} \delta \gamma^{-7 / 2}+C_{2} \gamma^{1 / 2} .
$$

The optimal convergence rate obtained is $\left\|f-f^{\alpha, \delta}\right\| \sim \delta^{1 / 8}$ with the choice $\gamma=\delta^{1 / 8}$.

It should be noted that the convergence rate obtained is very low. Yet it can not be improved with a Tikhonov regularization for CTF: with the bounds for the norm of $\widetilde{A}_{\gamma} A-I$ for the Tikhonov method (36) and for the norm of $R_{\gamma}$ (86), (92) shows that the convergence of $I-\widetilde{U} U$ towards zero is not achieved. 


\section{Conclusion}

In this paper, we have investigated the convergence of regularization methods for phase retrieval and phase contrast tomography. For the phase retrieval problem, the Tikhonov regularization, quasiparticule approach, and regularization with mollification methods have been studied and the optimal convergence rates have been estimated. The phase contrast tomography problem has also been studied. The inverse of the Radon operator is regularized with a rectangular window for the Riesz kernel or with the approximate inverse method based on mollifiers. The a priori convergence rate for the combined inversion of the Radon and phase retrieval operators is estimated for each approach.

\section{Conflict of Interests}

The author declares that there is no conflict of interests regarding the publication of this paper.

\section{References}

[1] A. Momose, T. T. Takeda, Y. Itai, A. Yoneyama, and K. Hirano, "Phase-contrast tomographic imaging using an X-ray interferometer," Journal of Synchrotron Radiation, vol. 5, no. 3, pp. 309314, 1998.

[2] G. R. Davis and F. S. L. Wong, "X-ray microtomography of bones and teeth," Physiological Measurement, vol. 17, no. 3, pp. 121-146, 1996.

[3] M. Salomé, F. Peyrin, P. Cloetens et al., "A synchrotron radiation microtomography system for the analysis of trabecular bone samples," Medical Physics, vol. 26, no. 10, pp. 2194-2204, 1999.

[4] S. Nuzzo, F. Peyrin, P. Cloetens, J. Baruchel, and G. Boivin, "Quantification of the degree of mineralization of bone in three dimensions using synchrotron radiation microtomography," Medical Physics, vol. 29, no. 11, pp. 2672-2681, 2002.

[5] S. Bayat, L. Apostol, E. Boller, T. Brochard, and F. Peyrin, "In vivo imaging of bone micro-architecture in mice with 3D synchrotron radiation micro-tomography," Nuclear Instruments and Methods in Physics Research, Section A: Accelerators, Spectrometers, Detectors and Associated Equipment, vol. 548, no. 1-2, pp. 247-252, 2005.

[6] C. Chappard, A. Basillais, L. Benhamou et al., "Comparison of synchrotron radiation and conventional $\mathrm{x}$-ray microcomputed tomography for assessing trabecular bone microarchitecture of human femoral heads," Medical Physics, vol. 33, no. 9, pp. 35683577, 2006.

[7] P. Cloetens, P. W. Ludwig, J. Baruchel et al., "Holotomography: quantitative phase tomography with micrometer resolution using hard synchrotron radiation x rays," Applied Physics Letters, vol. 75, no. 19, pp. 2912-2914, 1999.

[8] M. Langer, P. Cloetens, A. Pacureanu, and F. Peyrin, "X-ray inline phase tomography of multimaterial objects," Optics Letters, vol. 37, no. 11, pp. 2151-2153, 2012.

[9] P. Cloetens, R. Barrett, J. Baruchel, J.-P. Guigay, and M. Schlenker, "Phase objects in synchrotron radiation hard x-ray imaging," Journal of Physics D: Applied Physics, vol. 29, no. 1, pp. 133-146, 1996.

[10] M. Langer, P. Cloetens, J.-P. Guigay, and F. Peyrin, "Quantitative comparison of direct phase retrieval algorithms in in-line phase tomography," Medical Physics, vol. 35, no. 10, pp. 4556-4565, 2008.

[11] M. Langer, P. Cloetens, and F. Peyrin, "Regularization of phase retrieval with phase-attenuation duality prior for 3-D holotomography," IEEE Transactions on Image Processing, vol. 19, no. 9, pp. 2428-2436, 2010.

[12] V. Davidoiu, B. Sixou, M. Langer, and F. Peyrin, "Non-linear iterative phase retrieval based on Frechet derivative," Optics Express, vol. 19, no. 23, pp. 22809-22819, 2011.

[13] J. P. Guigay, M. Langer, R. Boistel, and P. Cloetens, "A mixed transfer function and transport of intensity approach for phase retrieval in the Fresnel region," Optics Letters, vol. 32, no. 12, pp. 1617-1619, 2007.

[14] T. E. Gureyev and K. A. Nugent, "Phase retrieval with the transport-of-intensity equation. II. Orthogonal series solution for nonuniform illumination," Journal of the Optical Society of America A: Optics and Image Science, and Vision, vol. 13, no. 8, pp. 1670-1682, 1996.

[15] T. E. Gureyev, "Composite techniques for phase retrieval in the Fresnel region," Optics Communications, vol. 220, no. 1-3, pp. 49-58, 2003.

[16] K. A. Nugent, "Coherent methods in the X-ray sciences," Advances in Physics, vol. 59, no. 1, pp. 1-99, 2010.

[17] D. Paganin, S. C. Mayo, T. E. Gureyev, P. R. Miller, and S. W. Wilkins, "Simultaneous phase and amplitude extraction from a single defocused image of a homogeneous object," Journal of Microscopy, vol. 206, no. 1, pp. 33-40, 2002.

[18] D. Paganin, Coherent X-Ray Optics, Oxford University Press, New York, NY, USA, 2006.

[19] H. W. Engl, M. Hanke, and A. Neubauer, Regularization of Inverse Problems, vol. 375 of Mathematics and Its Applications, Kluwer Academic Publishers, Dordrecht, The Netherlands, 1996.

[20] M. Born and E. Wolf, Principles of Optics, Cambridge University Press, New York, NY, USA, 1997.

[21] J. W. Goodman, Introduction to Fourier Optics, Roberts and Company, Greenwood Village, Colo, USA, 2005.

[22] J. Moosmann, R. Hofmann, and T. Baumbach, "Single-distance phase retrieval at large phase shifts," Optics Express, vol. 19, no. 13, pp. 12066-12073, 2011.

[23] R. Hofmann, J. Moosmann, and T. Baumbach, "Criticality in single-distance phase retrieval," Optics Express, vol. 19, no. 27, pp. 25881-25890, 2011.

[24] J. Moosmann, R. Hofmann, A. V. Bronnikov, and T. Baumbach, "Nonlinear phase retrieval from single-distance radiograph," Optics Express, vol. 18, no. 25, pp. 25771-25785, 2010.

[25] T. E. Gureyev, D. M. Paganin, G. R. Myers, Y. I. Nesterets, and S. W. Wilkins, "Phase-and-amplitude computer tomography," Applied Physics Letters, vol. 89, no. 3, Article ID 034102, 2006.

[26] F. Natterer, The Mathematics of Computerized Tomography, John Wiley \& Sons, New York, NY, USA, 1986.

[27] R. A. Adams, Sobolev Spaces, Academic Press, New York, NY, USA, 1975.

[28] B. Sixou, V. Davidoiu, M. Langer, and F. Peyrin, "Absorption and phase retrieval with Tikhonov and joint sparsity regularizations," Inverse Problems and Imaging, vol. 7, no. 1, pp. 267-282, 2013.

[29] E. M. Stein, Harmonic Analysis: Real Variables Methods, Orthogonality, and Oscillatory Integrals, Princeton University Press, 1993. 
[30] E. M. Stein, Singular Integrals and Differentiability Properties of Functions, Princeton University Press, 1970.

[31] J. Alvarez and M. Guzmán-Partida, “The $S$ ' -convolution with singular kernels in the Euclidean case and the product domain case," Journal of Mathematical Analysis and Applications, vol. 270, no. 2, pp. 405-434, 2002.

[32] E. T. Quinto, "Singularities of the X-ray transform and limited data tomography in $\mathbb{R}^{2}$ and $\mathbb{R}^{3}$," SIAM Journal on Mathematical Analysis, vol. 24, no. 5, pp. 1215-1225, 1993.

[33] T. Schuster, The Method of Approximate Inverse: Theory and Application, vol. 1906 of Lecture Notes in Mathematics, Springer, Berlin, Germany, 2007.

[34] K. Bingham, The mathematics of local X-ray tomography [Ph.D. thesis], 1998. 


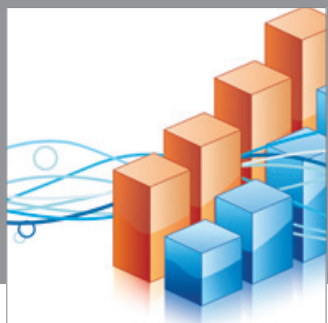

Advances in

Operations Research

mansans

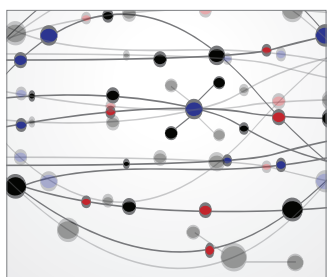

The Scientific World Journal

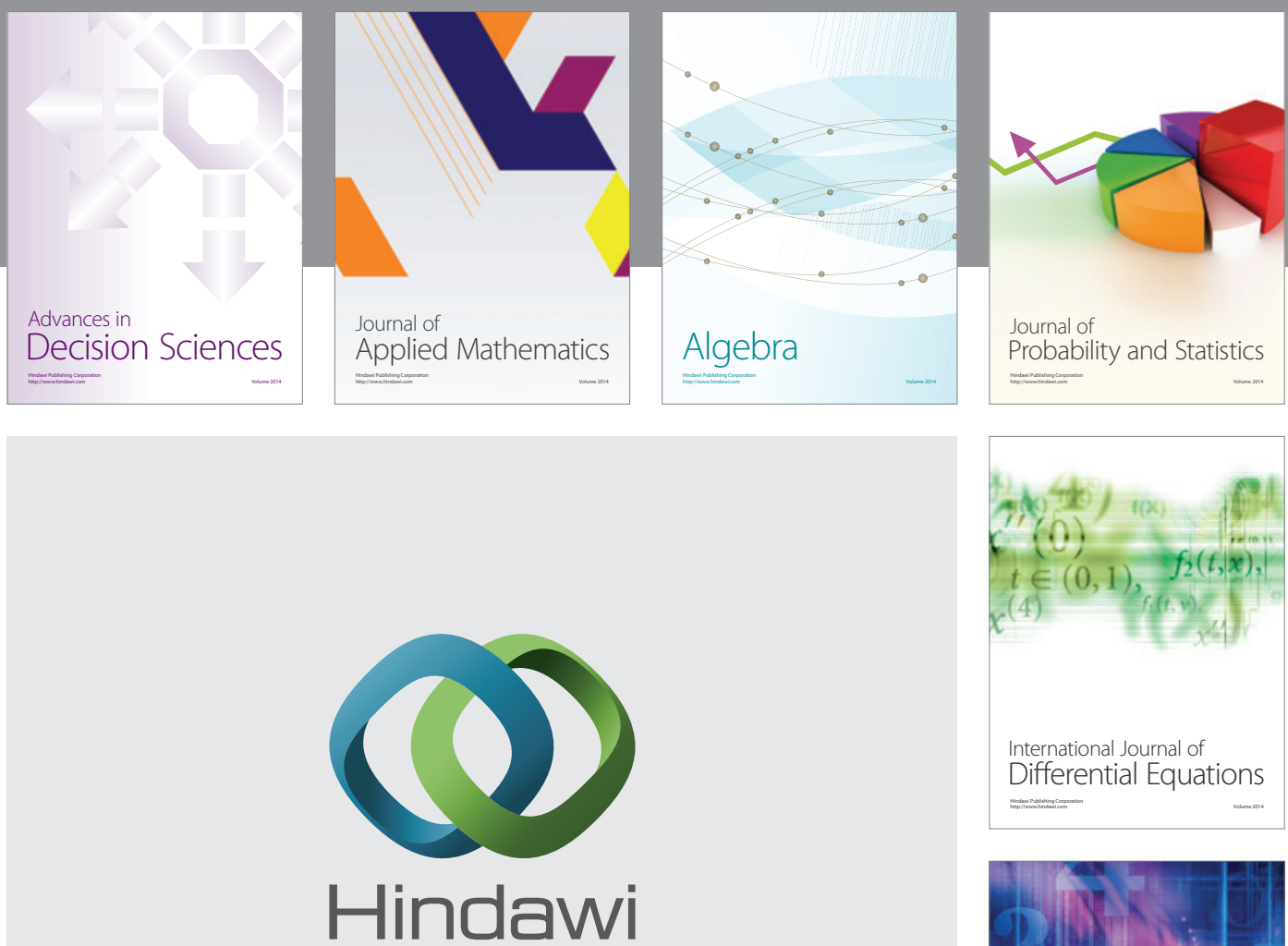

Submit your manuscripts at http://www.hindawi.com
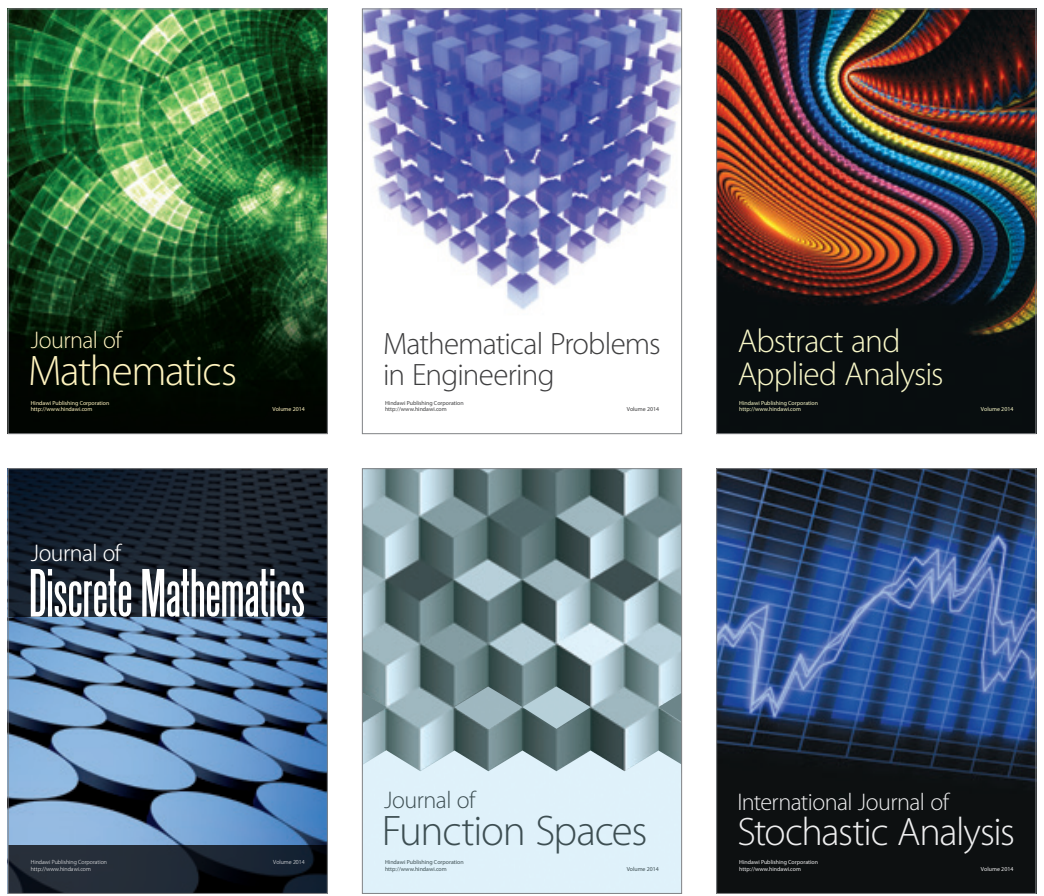

Journal of

Function Spaces

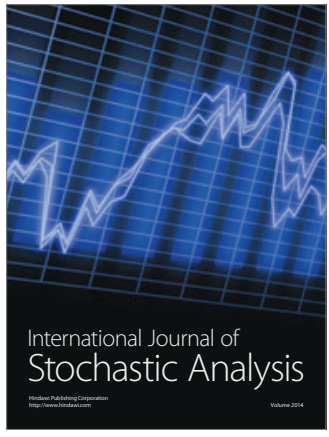

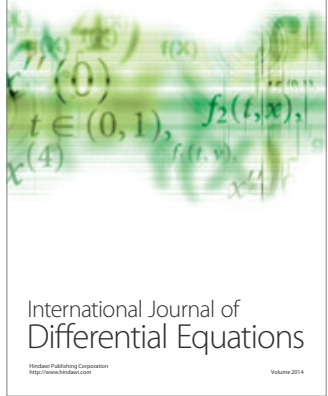
